\title{
Behavioural weight-loss intervention in high-risk patients
}

Targeted behavioural weight-loss intervention might benefit patients with serious mental illnesses, say researchers in the New England Journal of Medicine.

Overweight and obesity are prevalent among individuals with serious mental illnesses, such as schizophrenia, bipolar disorder and major depression. Nevertheless, these individuals are often excluded from weight-loss trials owing to concerns about adherence to lifestyle intervention programs as a result of a high frequency of psychiatric symptoms and impaired cognitive function, particularly in patients with schizophrenia.

In a randomized controlled trial including adults with serious mental illnesses, Daumit et al. assessed the efficacy of an 18-month behavioural weight-loss intervention, consisting of tailored individual and group weightmanagement sessions and group exercise sessions. The lifestyle intervention was based on programs originally designed for and shown to be effective in the general population. However, Daumit and colleagues tailored the intervention to address deficits in memory and executive function, for example, by simplifying and dividing information into small components and frequent repetition.

Study participants consisted of 291 adults with a mean age of 45.3 years who were overweight or obese (mean BMI $36.3 \mathrm{~kg} / \mathrm{m}^{2}$, mean weight $102.7 \mathrm{~kg}$ ). The patients had been recruited from 10 community psychiatric rehabilitation outpatient programs. Follow-up assessments occurred at 6,12 and 18 months. A total of $58.1 \%$ of trial participants had schizophrenia or a schizoaffective disorder, 22.0\% had bipolar disorder and $12.0 \%$ had major depression. Data on weight were obtained from 279 participants at the end of the study period.

Compared with the control group, who had received standard nutrition and physical-activity information at baseline and health classes on weight-unrelated content (for example, on cancer screening) every 4 months, the intervention group exhibited increased progressive weight loss over the study period. Weight differed significantly between the two groups at each follow-up appointment. At 18 months, the mean change in weight from baseline was $-0.2 \mathrm{~kg}$ in the control group compared with $-3.4 \mathrm{~kg}$ in the intervention group. The net difference in change in BMI was -1.1 for those undergoing lifestyle intervention compared with the control group. Moreover, almost $38 \%$ of participants in the intervention group lost $\geq 5 \%$ of their initial weight compared with $23 \%$ in the control group. No differences in adverse events were reported between the two groups.

Daumit and co-workers conclude that a lifestyle intervention program adapted to meet the needs of patients with serious mental illnesses shows great promise for weight reduction in this high-risk population.

Linda Koch

Original article Daumit, G. L. et al. A behavioral weight-loss intervention in persons with serious mental illness. N. Engl. J. Med. doi:10.1056/NEJMoa1214530 